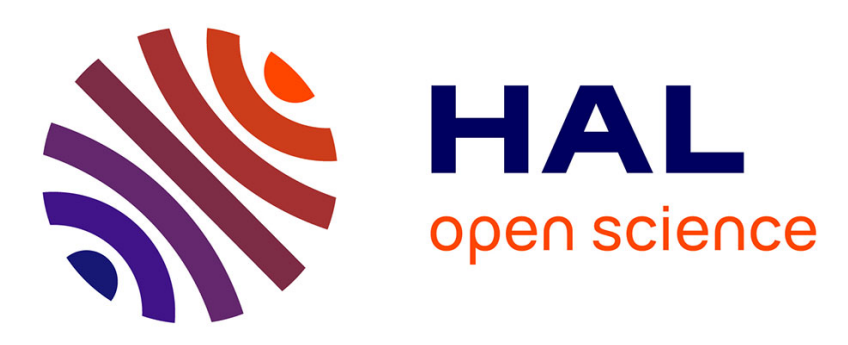

\title{
Statistical description of turbulent particle-laden flows in the very dilute regime using the Anisotropic Gaussian Moment Method
}

\author{
Macole Sabat, Aymeric Vié, Adam Larat, Marc Massot
}

\section{To cite this version:}

Macole Sabat, Aymeric Vié, Adam Larat, Marc Massot. Statistical description of turbulent particleladen flows in the very dilute regime using the Anisotropic Gaussian Moment Method. International Journal of Multiphase Flow, 2019, 112, pp.243-257. 10.1016/j.ijmultiphaseflow.2018.10.004 . hal01886835

\section{HAL Id: hal-01886835 \\ https://hal.science/hal-01886835}

Submitted on 4 Oct 2018

HAL is a multi-disciplinary open access archive for the deposit and dissemination of scientific research documents, whether they are published or not. The documents may come from teaching and research institutions in France or abroad, or from public or private research centers.
L'archive ouverte pluridisciplinaire $\mathbf{H A L}$, est destinée au dépôt et à la diffusion de documents scientifiques de niveau recherche, publiés ou non, émanant des établissements d'enseignement et de recherche français ou étrangers, des laboratoires publics ou privés. 


\title{
Statistical description of turbulent particle-laden flows in the very dilute regime using the Anisotropic Gaussian Moment Method
}

\author{
Macole Sabat ${ }^{\mathrm{a}, \mathrm{d}}$, Aymeric Viéa, ${ }^{\mathrm{a}, *}$, Adam Larat $^{\mathrm{a}, \mathrm{b}}$, Marc Massot $^{\mathrm{c}}$ \\ ${ }^{a}$ Laboratoire EM2C UPR 288, CNRS, CentraleSupélec, Université Paris-Saclay, 3, rue \\ Joliot-Curie 91192 Gif-sur-Yvette cedex France \\ ${ }^{b}$ Fédération de Mathématiques de l'Ecole Centrale Paris, CNRS, 3, rue Joliot-Curie 91192 \\ Gif-sur-Yvette cedex France \\ ${ }^{c}$ Centre de Mathématiques Appliquées, Ecole polytechnique, CNRS, Université \\ Paris-Saclay, Route de Saclay, 91128 Palaiseau Cedex, France \\ ${ }^{d}$ Mechanical Engineering Department, University of Balamand, Kalhat, Al Kurah, Lebanon
}

\begin{abstract}
The present work aims at investigating the ability of a Kinetic-Based Moment Method (KBMM) to reproduce the statistics of turbulent particle-laden flows using the Anisotropic Gaussian (AG) closure. This method is the simplest KBMM member that can account for Particle Trajectory Crossing (PTC) properly with a well-posed mathematical structure [1]. In order to validate this model further, we investigate here $3 \mathrm{D}$ turbulent flows that are more representative of the mixing processes, which occurs in realistic applications. The chosen configuration is a 3D statistically-stationary Homogeneous Isotropic Turbulence (HIT) loaded with particles in a very dilute regime. The analysis focuses on the description of the first three lowest order moments of the particulate flow: the number density, the Eulerian velocity and the internal energy. A thorough numerical study on a large range of particle inertia allows us to show that the AG closure extends the ability of the Eulerian models to correctly reproduce the particle dynamics up to a Stokes number based on the Eulerian turbulence macro-scale equal to one, but also highlights the necessity of high-order numerical schemes to reach mesh convergence, especially for the number density field.
\end{abstract}

\footnotetext{
* Corresponding author

Email address: aymeric.vie@centralesupelec.fr (Aymeric Vié)
} 
Keywords: Disperse phase, Eulerian models, Anisotropic Gaussian, Particle Trajectory Crossing, Turbulence.

\section{Introduction}

In several industrial systems such as aeronautical combustors or fluidized beds, turbulent particle- or droplet-laden flows play a central role. Simulating such flows is still challenging because of the multi-scale character of the interactions between the gaseous carrier phase and the disperse phase. Several methods are available in the literature, ranging from the simulation at the particle scale [2] to the ensemble-averaged simulation of a full system [3].

The present work focuses on the prediction of the dynamics of particles subject to drag force with a turbulent carrier phase in the very dilute regime. In this context, the particles do not affect the carrier phase and the inter-particle collisions are neglected. A point-particle approximation is considered, for which particle-scale flow fluctuations are dissipated fast enough to be neglected. Under these assumptions, the statistics of the disperse phase, i.e. its Number Density Function (NDF), can be determined by a Generalized Population Balance Equation (GPBE). As stated in [4], a key aspect of this statistical description is the particle velocity dispersion, called Random Uncorrelated Motion (RUM) due to the Particles Trajectory Crossing (PTC). This velocity dispersion is typical for inertial particles that are weakly correlated to the local fluid velocity field.

Solving the PBE directly is not feasible in realistic configurations due to the high dimensionality of the phase space. To circumvent this issue, moment methods can be used. This efficient strategy significantly reduces the computational cost by transporting moments of the NDF that only lie in the physical space. However, for a given moment set to be solved, unsolved higher order moments are required in order to close the moment equations. The closure must properly describe the velocity dispersion in order to reproduce the physics of the flow. In the literature, several closures can be found in the particulate flow community, but also in the rarefied gases community. They can be separated into two 
families depending of the type of approximations: the Algebraic-Closure-Based Moment Methods (ACBMM, [5, 6, 7, 8, 9]), which make assumptions on the structure and evolution of the moments, and the Kinetic-Based Moment Methods (KBMM, 10, 11, 12, 13, 1, 14, 15, ), which make assumptions directly on the shape of the NDF itself.

By essence, the two families share the objective of controlling moments up to a given order. However, ACBMM always require fewer moments to be solved because, when KBMM add an equation for any additional moments to be controlled, ACBMM can rely on an algebraic relationship. In [16], a first comparison between the 2- EEASM model [7] and the Anisotropic Gaussian (AG) model [1] has been performed, showing a comparable accuracy in a 2D turbulent configuration. Both methods were targeting to control the second order moments, but where AG has to solve the full set of second order moments (6 in 3D), 2 - EEASM model uses only one moment (the trace of the pressure matrix), leading to a reduced computational effort. However, the design of numerical methods is more difficult for ACBMM because of the presence of extra nonlinear terms preventing from a complete characterization of the mathematical structure. On the other hand, KBMM lead to hyperbolic or weakly-hyperbolic systems of equations [17, for which well-designed solvers exist in the literature (see [18, 19] and references therein for example).

Even if the AG model has already been validated in 2D and compared with a member of the ACBMM, it still has to be validated in 3D turbulent configurations in order to evaluate its ability to tackle realistic industrial configurations. This is the aim of the present paper.

In the following, the AG model is first presented along with the MonoKinetic model (MK): a model only accurate for small inertia particles [20. Numerical methods are also briefly mentioned, while a more detailed description is provided in Appendix B. Next, we aim at showing that the second-order AG model can be viewed as an extension of the first-order MK model. To this purpose, a 3D turbulent configuration is presented and the results obtained with the AG and MK models are compared to a Lagrangian statistically-converged reference. The 
numerical study is performed in an as extensive manner as possible, by sweeping a large range of the disperse regimes and looking at numerous representative quantities to assess the strengths and weaknesses of the AG Eulerian model. By this thorough numerical study, we assess the ability of the AG model to recover the right statistics for a specified range of particle inertia.

\section{Statistical modelling of particle-laden flows}

The present work relies on the following assumptions:

- Point-particle approximation [21]: particles are considered to be much smaller than any of the carrier phase scales.

- Negligible mass loading and volume fraction: the particles load does not affect the carrier phase. The two phases are coupled only one-way: the carrier phase feeds the disperse phase with energy, but the particles retroaction is neglected.

- Very dilute regime: the particles mean free path is much greater than any dimension of the problem. The Knudsen number is considered to be infinite and thus, inter-particles collisions are neglected.

- Large density ratio between particle and gas phases: the drag force is the only force acting on the particles.

- Fixed-size monodisperse spherical particles: there is no need to track the distribution in size.

- Stokes drag: particules Reynolds number does not affect their relaxation time toward the underlying carrier velocity field.

In this framework, the NDF, $f(t, \overrightarrow{\boldsymbol{x}}, \overrightarrow{\boldsymbol{c}})$, of the disperse phase is described by the following GPBE:

$$
\partial_{t} f+\partial_{\overrightarrow{\boldsymbol{x}}} \cdot(\overrightarrow{\boldsymbol{c}} f)+\partial_{\overrightarrow{\boldsymbol{c}}} \cdot\left(\frac{\overrightarrow{\boldsymbol{u}}_{g}-\overrightarrow{\boldsymbol{c}}}{\tau_{d}} f\right)=0,
$$

where $\overrightarrow{\boldsymbol{c}}$ is the velocity of the particles in the phase space, $\overrightarrow{\boldsymbol{u}}_{g}$ is the gas velocity and $\tau_{d}$ is the characteristic relaxation time of the particles. 


\subsection{Eulerian method of moments}

After multiplying equation (1) by $c_{1}^{i} c_{2}^{j} c_{3}^{k}$ and integrating over the phase space, one gets a system of moment equations. In a three-dimensional physical space, the general equation on the velocity moments is:

$$
\partial_{t} M_{i, j, k}+\partial_{\overrightarrow{\boldsymbol{x}}} \cdot\left(\begin{array}{c}
M_{i+1, j, k} \\
M_{i, j+1, k} \\
M_{i, j, k+1}
\end{array}\right)=\frac{1}{\tau_{d}}\left(\overrightarrow{\boldsymbol{u}}_{g} \cdot\left(\begin{array}{c}
i M_{i-1, j, k} \\
j M_{i, j-1, k} \\
k M_{i, j, k-1}
\end{array}\right)-(i+j+k) M_{i, j, k}\right),
$$

where the general $(i+j+k)^{t h}$ order moment in velocity is:

$$
M_{i, j, k}=\int c_{1}^{i} c_{2}^{j} c_{3}^{k} f \mathrm{~d} c_{1} \mathrm{~d} c_{2} \mathrm{~d} c_{3} .
$$

This system is not closed: actually for every set of moments of order $N$ which contains the moments of order $(i+j+k) \leq N$, moments of order $N+1$ are needed to describe the higher order fluxes in physical space: $M_{i, j, k}$ where $i+j+k=N+1$.

In order to close the moment system (2), one needs to provide:

- the moment set of order $N$ :

$$
\left\{M_{i, j, k}, i+j+k \leq N\right\},
$$

- a closure relationship which allows to model the unknown fluxes. In the Kinetic Based Moment Methods (KBMM), this is done by keeping the link between the kinetic level and the macroscopic level, at the price of the reconstruction of the NDF, which is generally supposed to have a given shape.

The choice of the presumed shape of the NDF is of paramount importance for a KBMM. It can be driven by the following guidelines:

- a one-to-one mapping between the moments and the parameters of the NDF is essential to ensure the well-posedness of the system of equations; 
- an appropriate shape can also guarantee that the final moment system is hyperbolic and has an entropic structure, usually relying on hydrodynamic equilibrium assumption as in the Levermore hierarchy [22];

- the shape can also be chosen based on the physics of the problem in sight, which can for instance suggest an equilibrium distribution (such as a Maxwellian distribution in the case of collision-driven particulate flows);

- peculiar attention must be given to the border of the moment space (zero pressure, zero density), to avoid numerical issues when dealing with transition between regions at the border and regions inside the moment space.

\subsection{Monokinetic model}

When no PTC occur, the disperse phase has a unique deterministic velocity at each time-space point $(t, \overrightarrow{\boldsymbol{x}})$. Consequently, the velocity distribution is monokinetic:

$$
f(t, \overrightarrow{\boldsymbol{x}}, \overrightarrow{\boldsymbol{c}})=n(t, \overrightarrow{\boldsymbol{x}}) \delta(\overrightarrow{\boldsymbol{c}}-\overrightarrow{\boldsymbol{u}}(t, \overrightarrow{\boldsymbol{x}})),
$$

where $n(t, \overrightarrow{\boldsymbol{x}})$ is the total number density of the disperse phase, and $\overrightarrow{\boldsymbol{u}}(t, \overrightarrow{\boldsymbol{x}})$ the Eulerian velocity. In this specific case, the system of moments closes at first order, since the pressure tensor, its second order centered-moment, is null: $\mathbf{P}=\int(\overrightarrow{\boldsymbol{c}}-\overrightarrow{\boldsymbol{u}}(t, \overrightarrow{\boldsymbol{x}})) \otimes(\overrightarrow{\boldsymbol{c}}-\overrightarrow{\boldsymbol{u}}(t, \overrightarrow{\boldsymbol{x}})) f(t, \overrightarrow{\boldsymbol{x}}, \overrightarrow{\boldsymbol{c}}) d \overrightarrow{\boldsymbol{c}}=\mathbf{0}$. Therefore, we get the following weakly-hyperbolic system, identical to the Pressureless Gas Dynamics (PGD) system [23, 24]:

$$
\left\{\begin{array}{l}
\partial_{t} n+\partial_{\overrightarrow{\boldsymbol{x}}} \cdot(n \overrightarrow{\boldsymbol{u}})=0, \\
\partial_{t}(n \overrightarrow{\boldsymbol{u}})+\partial_{\overrightarrow{\boldsymbol{x}}} \cdot(n \overrightarrow{\boldsymbol{u}} \otimes \overrightarrow{\boldsymbol{u}})=\frac{n\left(\overrightarrow{\boldsymbol{u}}_{g}-\overrightarrow{\boldsymbol{u}}\right)}{\tau_{d}} .
\end{array}\right.
$$

The MK model correctly reproduces the formation of depletion and accumulation regions obtained for small Stokes numbers and dedicated numerical schemes can be designed to properly account for such hypercompressible dynamics [20. However, when PTC occur for moderate to high inertial particles, the velocity dispersion cannot be captured by the single Dirac $\delta$-function used for 
the velocity distribution. Instead, at each PTC location, unphysical $\delta$-shocks are generated, and these are highly constraining from the numerical point of view. Another constraint of this model is that it does not conserve the total kinetic energy of the disperse phase [23, 25].

\subsection{Anisotropic Gaussian model}

To reproduce the velocity dispersion induced by the PTC, we consider the AG closure, which is a second order model of the KBMM family:

$$
f(t, \overrightarrow{\boldsymbol{x}}, \overrightarrow{\boldsymbol{c}})=n(t, \overrightarrow{\boldsymbol{x}}) \mathcal{N}(\overrightarrow{\boldsymbol{c}}-\overrightarrow{\boldsymbol{u}}(t, \overrightarrow{\boldsymbol{x}}), \boldsymbol{\Sigma}(t, \overrightarrow{\boldsymbol{x}}))
$$

where $\mathcal{N}$ is a joint Gaussian distribution centered in $\overrightarrow{\boldsymbol{u}}$ and of covariance matrix $\boldsymbol{\Sigma}=\left(\sigma_{i j}\right)$ in the space of dimension $N_{d}$ :

$$
\mathcal{N}(\overrightarrow{\boldsymbol{c}}-\overrightarrow{\boldsymbol{u}}, \boldsymbol{\Sigma})=\frac{|\boldsymbol{\Sigma}|^{-1 / 2}}{(2 \pi)^{N_{d} / 2}} \exp \left(-\frac{1}{2}(\overrightarrow{\boldsymbol{c}}-\overrightarrow{\boldsymbol{u}})^{T} \boldsymbol{\Sigma}^{-1}(\overrightarrow{\boldsymbol{c}}-\overrightarrow{\boldsymbol{u}})\right) .
$$

This closure was inspired by the work of [26] in the context of out-of-equilibrium rarefied gas dynamics and was called the 10-moment closure, and is the first member of the Levermore hierarchy [22]. In the rarefied gases context, the good mathematical properties of this model at the kinetic and at the moment levels was highlighted in [27, 28] and a numerical approximation was proposed by [29] and [30.

The resulting system of moments reads:

$$
\left\{\begin{array}{l}
\partial_{t} n+\partial_{\overrightarrow{\boldsymbol{x}}} \cdot(n \overrightarrow{\boldsymbol{u}})=0, \\
\partial_{t}(n \overrightarrow{\boldsymbol{u}})+\partial_{\overrightarrow{\boldsymbol{x}}} \cdot(n \overrightarrow{\boldsymbol{u}} \otimes \overrightarrow{\boldsymbol{u}}+\mathbf{P})=\frac{n\left(\overrightarrow{\boldsymbol{u}}_{g}-\overrightarrow{\boldsymbol{u}}\right)}{\tau_{d}}, \\
\partial_{t}(n \mathbf{E})+\partial_{\overrightarrow{\boldsymbol{x}}} \cdot((n \mathbf{E}+\mathbf{P}) \vee \overrightarrow{\boldsymbol{u}})=\frac{n\left(\overrightarrow{\boldsymbol{u}}_{g} \vee \overrightarrow{\boldsymbol{u}}-2 \mathbf{E}\right)}{\tau_{d}},
\end{array}\right.
$$

where $\vee$ denotes the symmetric tensor outer product 1 the total energy tensor

\footnotetext{
${ }^{1}$ The symmetric tensor outer product acts on a symmetric $k$-tensor and a symmetric $l$ tensor by symmetrizing their $(k+l)$-order outer product tensor, see [26].
} 
$\mathbf{E}$ is given by $\mathbf{E}=\frac{1}{2} \overrightarrow{\boldsymbol{u}} \otimes \overrightarrow{\boldsymbol{u}}+\frac{\mathbf{P}}{2 n}$ and the anisotropic pressure tensor is given by the equation of state $\mathbf{P}=n \boldsymbol{\Sigma}$. The resulting system is hyperbolic and admits entropies [31]. This model was introduced for the first time for the modeling of the disperse phase in the work of $[1]$.

\subsection{Realizable numerical schemes}

An important requirement for the moment methods is to preserve the realizability of the moment set, i.e. the fact that the moment set is the set of moments of a positive NDF. Because the closure of the moment set is obtained through assumptions on the NDF itself, the derived systems of moments preserve the realizability of the moment set at the continuous level. When it comes to solve the moment system using a given time and space discretization, introducing numerical errors, it is not straightforward to maintain the realizability of the moments at the discrete level, especially since we want to cover the whole moment space, including its frontier (zero density, zero pressure, ...).

In the present work, the two systems of interest are solved using numerical methods expressly developed to ensure realizability and stability, as well as high accuracy: a dedicated second order MUSCL-HLL scheme.

For the AG model, the MUSCL-HLL scheme developed in [1] has been extended to the 3D case. It is of second-order in time and space, also preserves realizability of the moment set and behaves well at moment space boundaries. More details are given in Appendix B.

It is important to note that for the Monokinetic approach, a second-order Kinetic Scheme 32] can also be used. The realizability properties of this latter are inherited from a linear and conservative reconstruction of the central mo-

ments $n$ and $u$, and an exact evolution in time of the reconstructed NDF at the kinetic level.

\section{Numerical setup: 3D Forced Homogeneous Isotropic Turbulence}

The configuration of interest is based on a 3D Forced Homogeneous Isotropic Turbulence (FHIT) for the carrier phase, solved in a cubic domain, with periodic 
boundary conditions. This classical academic test case, auto-similar in terms of Reynolds number, is essential for the validation of the disperse phase models, since it represents one of the canonical samples of the physics faced in industrial applications. These simulations are carried out at a low Reynolds number. However, let us emphasize here that the evaluation and the application of the Anisotropic Gaussian model is not limited to low turbulence flows.

All the values of the variables presented in this work are dimensionless:

$$
u^{*}=\frac{u}{u_{r e f}}, \quad t^{*}=\frac{t}{t_{r e f}}, \quad L^{*}=\frac{L}{L_{r e f}},
$$

and the normalizing Reynolds number is $\operatorname{Re}_{0}=u_{\text {ref }} L_{r e f} / \nu=10^{3}$.

\subsection{Velocity field and general properties}

The FHIT is solved using the ASPHODELE code [33, which solves the Navier-Stokes equations in the low-Mach number limit.

The initial turbulence field is generated using an isotropic random mode generation and inverse Fourier transform in a cubic domain of size $5^{3}$ using a $128^{3}$ mesh. The energy spectrum function introduced in 34 is used as the model spectrum:

$$
E_{m}(\kappa)=C\langle\varepsilon\rangle^{2 / 3} \kappa^{-5 / 3} f_{L}(\kappa L) f_{\eta}(\kappa \eta),
$$

with:

$$
\begin{gathered}
f_{L}(\kappa L)=\left(\frac{\kappa L}{\left[(\kappa L)^{2}+c_{L}\right]^{1 / 2}}\right)^{5 / 3+p_{0}}, \\
f_{\eta}(\kappa \eta)=\exp \left(-\beta\left[\left((\kappa \eta)^{4}+c_{\eta}^{4}\right)^{1 / 4}-c_{\eta}\right]\right),
\end{gathered}
$$

where $\eta=\left(\frac{\nu^{3}}{\langle\varepsilon\rangle}\right)^{1 / 4}$ is the Kolmogorov length scale given as a function of the carrier phase viscosity $\nu$ and the mean dissipation rate of turbulent kinetic energy $\langle\varepsilon\rangle$. In addition, the length scale $L=\frac{\langle\mathrm{TKE}\rangle^{3 / 2}}{\langle\varepsilon\rangle}$ characterizes the large eddies, where $\langle\mathrm{TKE}\rangle$ is the mean turbulent kinetic energy. The initial parameters of the spectrum are $C=1.5, \beta=5.2$ and $p_{0}=4$ [34], $c_{L}=0.019,\langle\mathrm{TKE}\rangle=0.00667$, $c_{\eta}=0.051, L=4$ and $\eta=0.027$. 
The turbulent characteristics of the statistically-stationary HIT, including the mean turbulent kinetic energy $(\langle\mathrm{TKE}\rangle)$, the dissipation $\langle\varepsilon\rangle$, the Kolmogorov time and length scales (time $\tau_{K}$ and length $\eta$ ), the Lagrangian integral time scale $\tau_{L}$, the Eulerian eddy turn-over time $\tau_{e d d y}$, the longitudinal integral length scale $L_{11}$, and the turbulent Reynolds number $\operatorname{Re}=u_{g, r m s} L_{11} / \nu$, where $u_{g, r m s}$ is the gas velocity fluctuations, are given in Table 1 .

Table 1: Turbulence properties of the HIT.

\begin{tabular}{|c|c|c|c|c|c|c|c|}
\hline$\langle\mathrm{TKE}\rangle$ & $\langle\varepsilon\rangle$ & $\tau_{K}$ & $\eta$ & $\tau_{L}$ & $\tau_{\text {eddy }}$ & $L_{11}$ & $\operatorname{Re}$ \\
\hline 0.00667 & 0.000635 & 1.2545 & 0.03535 & 4.7905 & 9.7864 & 0.65217 & 43.46 \\
\hline
\end{tabular}

\subsection{Characteristic time scale of the fluid}

The Stokes number based on the Kolmogorov time scale $\mathrm{St}_{K}$ is classically used to characterize the occurrence of PTC, since it relies on the scales of the turbulence of the shortest characteristic time. This choice, although very common in DNS, can be tempered because the Kolmogorov time scale $\tau_{K}=\sqrt{\nu /\langle\varepsilon\rangle}$ represents an average time scale. Indeed, $\langle\varepsilon\rangle=\nu\left\langle 2 s_{i j} s_{i j}>\right.$ is obtained as an averaging over the spatial domain, where $s_{i j}=\frac{1}{2}\left(\frac{\partial u_{g, i}}{\partial x_{j}}+\frac{\partial u_{g, j}}{\partial x_{i}}\right)$. Therefore, shorter time scales may exist in the turbulence. To illustrate this possibility, the distribution of the characteristic time scales of the fluid $\tau_{\text {local }}=1 / \sqrt{s_{i j} s_{i j}}$ is plotted in Fig. 1. It shows the existence of short time scales that may generate PTC, even if $\mathrm{St}_{K}$ is below the critical value of 1 . Such a behaviour has already been reported in the literature, see for instance [35], and is of great interest for modelling purpose, as it could suggest that even low-Stokes number flows require the modelling of the second order moments.

\subsection{Eulerian and Lagrangian simulations for the particles}

Here we give details about the simulations that have been performed. For the initialisation, 100M lagrangian particles are randomly loaded in the domain following a homogeneous distribution. Eulerian simulations are also initially 


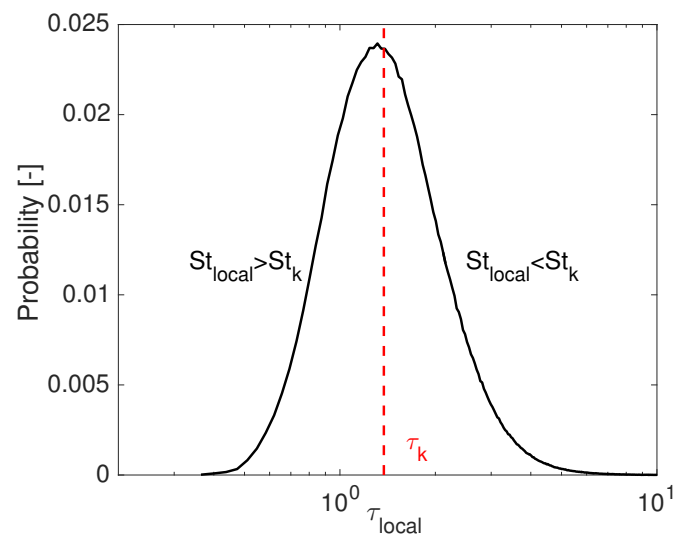

Figure 1: Distribution function of the local time scale of the gas velocity field for the FHIT at $t=40$ in black. The red line identifies the Kolmogorov time scale $\tau_{k}$.

set to a homogeneous distribution. For both types of simulations, all particles are at rest at the beginning. The simulated Stokes number are $\mathrm{St}_{K}=$ $[0.05,0.1,0.5,1.0,1.5,2.0,3.0,4.0,6.0,8.0,10.0,15.0]$. Simulations are performed up to $t=40$, except for $\mathrm{St}_{K} \geq 8$ that are simulated up to time $t=80$ in order to get relevant equilibrium statistics. Simulation times are reported in Table 2 for $\mathrm{St}_{K}=8.0$, for a computation up to $t=80$, on 512 intel Xeon E5-2690V3 processors.

\begin{tabular}{|c|c|c|c|c|c|c|c|c|c|}
\hline Simul. & Lag & \multicolumn{5}{|c|}{ MK } & \multicolumn{4}{c|}{ AG } \\
\hline Mesh & & $64^{3}$ & $128^{3}$ & $256^{3}$ & $512^{3}$ & $64^{3}$ & $128^{3}$ & $256^{3}$ & $512^{3}$ \\
\hline Time & $1 \mathrm{~h} 37 \mathrm{~m}$ & $33 \mathrm{~s}$ & $3 \mathrm{~m}$ & $32 \mathrm{~m}$ & $7 \mathrm{~h} 6 \mathrm{~m}$ & $54 \mathrm{~s}$ & $5 \mathrm{~m}$ & $57 \mathrm{~m}$ & $15 \mathrm{~h} 19 \mathrm{~m}$ \\
\hline
\end{tabular}

Table 2: Simulation time (wall time) for the particle solver using Lagrangian or Eulerian solvers. Simulations are performed on 512 Intel Xeon E5-2690V3 on the supercomputer Occigen (CINES, France).

\section{Results and discussions}

The results are presented in a sequential manner, comparing the AG and MK model to a Lagrangian reference. Eulerian quantities for the Lagrangian data 
are obtained using a box average based on the computational mesh. We first investigate the ability to accurately reproduce the number density field. Then, results on the Eulerian velocity are presented. Finally, the ability to predict the energy partition in the particles dynamics is evaluated.

\subsection{Number density field}

\subsubsection{Qualitative visualization}

The first level of validation consists in a qualitative comparison of the number density fields for three different Stokes numbers, see Fig. 2 .

For $\mathrm{St}_{K}=1$, the spatial structure to be captured is challenging from the numerical point of view: we have to deal with very high concentration in thin structures close to large depletion zones. In fact, the closer to the critical Stokes number, the greater the probability to generate $\delta$-shocks. At this particular Stokes number, AG and MK models provide the same global structure of the number density field, in agreement with the Lagrangian reference, despite the fact that Lagrangian solution presents thinner structures.

For $\mathrm{St}_{K}=3$, the underlying physics is expected to lead to significant PTC In this case, the MK model is not expected to capture the right dynamics of the particle-laden flow. This is clearly seen in Fig. 2, where particles accumulations are highly overestimated by the MK model compared to the Lagrangian and AG results. On the other hand, the AG model still captures the global structures, their sizes and does not overestimate the accumulations.

These conclusions still hold for $\mathrm{St}_{K}=8$, for which the MK model still overestimates the level of particles accumulation. The Lagrangian reference shows a lower level of accumulation compared to $\mathrm{St}_{K}=3$, and the $\mathrm{AG}$ model follows the same trend.

\subsubsection{Segregation}

In order to get a quantitative estimate of the level of accumulation and of depletion in the number density field, we investigate the segregation, which represents the spatial correlation of the number density field at a given length scale 

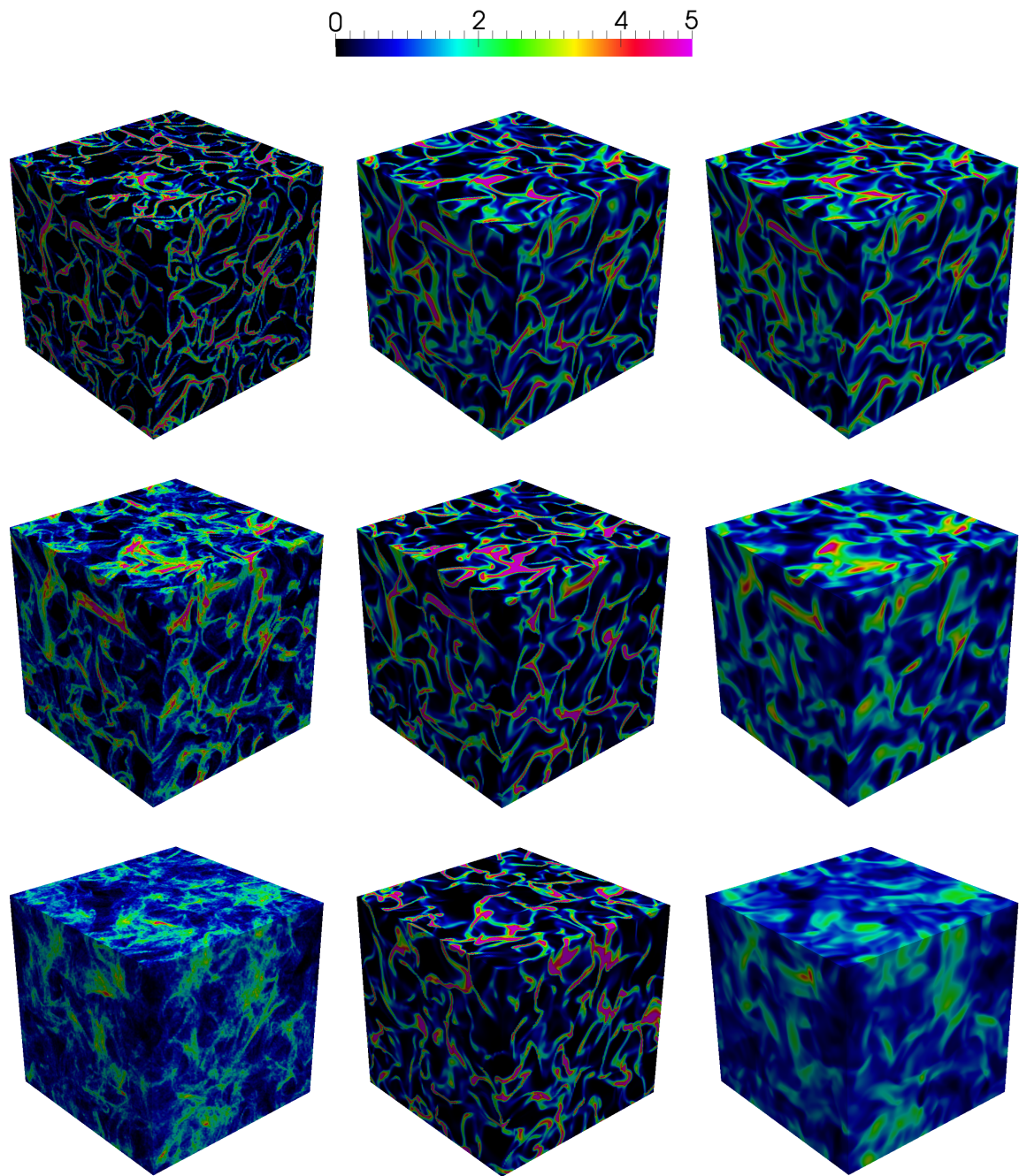

Figure 2: Number density field at $t=40$ on the $128^{3}$ mesh for $\mathrm{St}_{K}=1$ (top), $\mathrm{St}_{K}=3$ (center) and $\mathrm{St}_{K}=8$ (bottom), for the Lagrangian reference (left), the Mono-kinetic model (center) and the Anisotropic Gaussian model (right).

[36. This quantity is of paramount importance in the domain of combustion since it strongly affects the evolution of the mean vapour mixture fraction [33]. The segregation $G_{p p}^{\Delta}$ is given by the following equation:

$$
G_{p p}^{\Delta}=\frac{\left\langle n_{\Delta}^{2}\right\rangle}{\left\langle n_{\Delta}\right\rangle^{2}}
$$


where $\langle\cdot\rangle$ is the averaging operator over the whole domain, and $n_{\Delta}$ is the number density field projected onto a mesh of grid size $\Delta$, using a box filter. In the following, $\Delta$ is chosen to be equal to the largest mesh spacing used for the Eulerian simulation, i.e. $\Delta=\frac{L}{64}$. The time evolution of the segregation is plotted in Fig. 3 for three Stokes numbers and several mesh refinements.

For $\mathrm{St}_{K}=1$, both Eulerian models underestimate the segregation compared to the Lagrangian reference. The MK model gives higher segregation than the AG model, and is therefore closer to the Lagrangian segregation. When refining the mesh, both Eulerian models yield higher segregation and seem to "converge" to the Lagrangian reference. However for the $512^{3}$ mesh, the segregation of MK slightly overestimates the Lagrangian one for $t<20$, showing that this model is not converging to the Lagrangian result. This can be explained by the fact that the occurence of local strain rate higher than the average value used to compute $\tau_{k}$ can lead to PTC that cannot be handled correctly by the MK model.

In the case of moderately inertial particles $\mathrm{St}_{K}=3$, the segregation is overestimated by the MK model and diverges from the Lagrangian segregation curve, when refining the mesh. On the other hand, the segregation level for the AG model approaches to the Lagrangian one, when refining the mesh.

For inertial particles at $\mathrm{St}_{K}=8$, the segregation is still largely overestimated by the MK model, whereas the AG model is capturing the overall time evolution, and is getting closer to the Lagrangian reference as we refine the mesh. However, it should be noted that for long time $t=80$, the most refined solution of the AG model gives a slightly higher segregation than the Lagrangian reference.

From both high Stokes numbers, we clearly see the two main sources of "diffusion" of the Eulerian solutions, the numerical error and the model:

- the numerical error is present in both MK and AG simulations, and exhibits a first-order convergence in terms of segregation because the numerical schemes are necessarily switching to first order in accumulation zones and close to vacuum. The high sensitivity of Eulerian simulations to numerical error has already been evidenced in the literature [37, 5, 38, 39]; 
- for a given mesh, comparing AG and MK shows the "diffusive" effect of solving for an additional internal energy: the AG model always giving a lower segregation than the MK model.

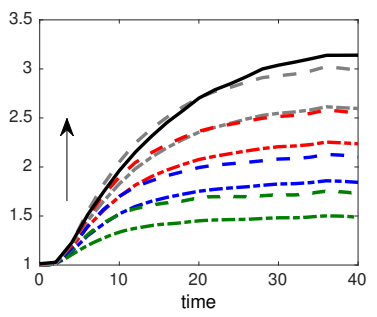

(a) $\mathrm{St}_{K}=1$

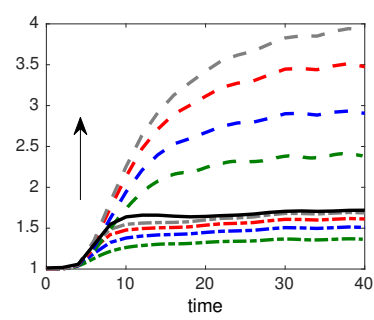

(b) $\mathrm{St}_{K}=3$

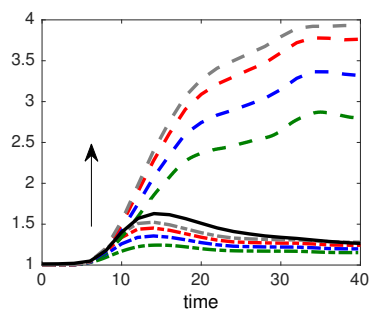

(c) $\mathrm{St}_{K}=8$

Figure 3: Time evolution of the segregation for the Lagrangian result (black solid line) and Eulerian results using $64^{3}$ (green), $128^{3}$ (blue), $256^{3}$ (red) and $512^{3}$ (grey) meshes, for $\mathrm{St}_{K}=1$, $\mathrm{St}_{K}=3$ and $\mathrm{St}_{K}=8$, for the MK model (dashed lines) and the AG model (dot-dashed lines). Arrows indicate increasing mesh sizes for Eulerian simulations.

\subsubsection{Distribution functions}

To further investigate the number density field, we look at its distribution in the physical domain for each Stokes number. At the critical Stokes number, $\mathrm{St}_{K}=1$, both Eulerian results approach the number density PDF of the Lagrangian result (Fig. 4) with mesh refinement. The number density PDF of the MK- $512^{3}$ result is however closer to the Lagrangian PDF than the most refined AG one. This kind of differences has also been evidenced on the literature, see [5. Nonetheless, the MK result might diverge from the Lagrangian one with even more refined meshes, since the PTC might occurs because of the smallest time scales of the turbulence.

For a moderately inertial disperse phase $\left(\mathrm{St}_{K}=3\right)$, see Fig. 5 , the number density PDF of the AG result approaches the Lagrangian one when refining the mesh, except for the underestimation of the vacuum zone. The MK number density distribution is not converging to the Lagrangian one when refining the mesh and gives a higher number of mesh cells where the number density is zero 


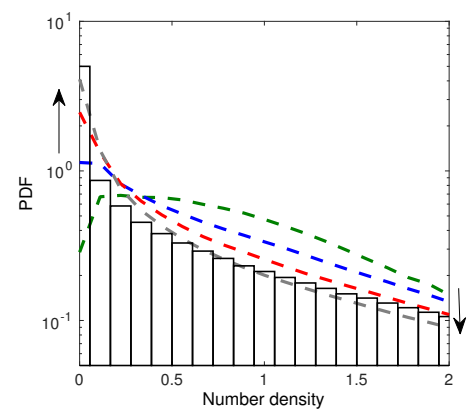

(a) MK

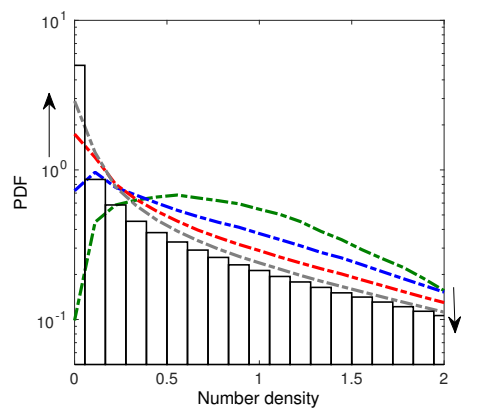

(b) AG

Figure 4: Number density PDF for the Lagrangian (black histogram), AG (dot-dashed lines) and MK (dashed lines) results on $64^{3}$ (green), $128^{3}$ (blue), $256^{3}$ (red) and $512^{3}$ (gray) meshes for $\mathrm{St}_{K}=1$. Arrows indicate increasing mesh sizes for Eulerian simulations.

or very small due to the singularities created by this model at each PTC.

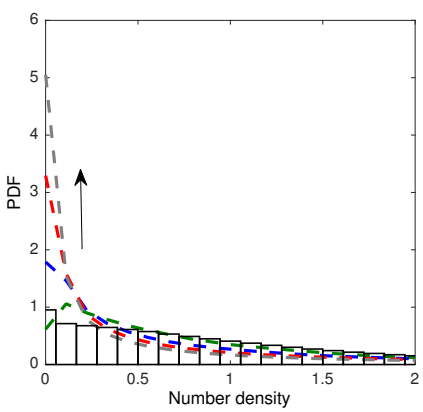

(a) MK

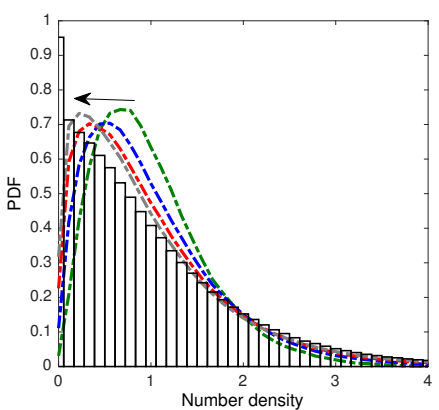

(b) AG

Figure 5: Number density PDF for the Lagrangian (black histogram), AG (dot-dashed lines) and MK (dashed lines) results on $64^{3}$ (green), $128^{3}$ (blue), $256^{3}$ (red) and $512^{3}$ (gray) meshes for $\mathrm{St}_{K}=3$. Arrows indicate increasing mesh sizes for Eulerian simulations.

At an even higher Stokes number $\left(\mathrm{St}_{K}=8\right)$, the distribution of the number density is plotted in Fig. 6, and the AG model is shown to properly capture the number density distribution compared to the Lagrangian reference. 


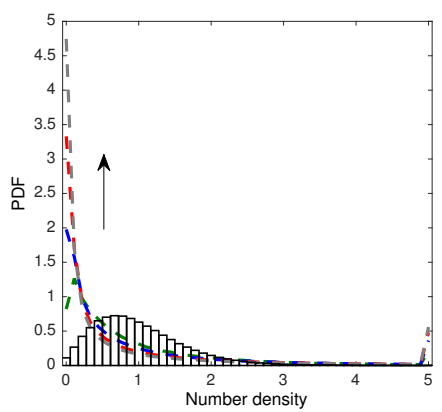

(a) MK

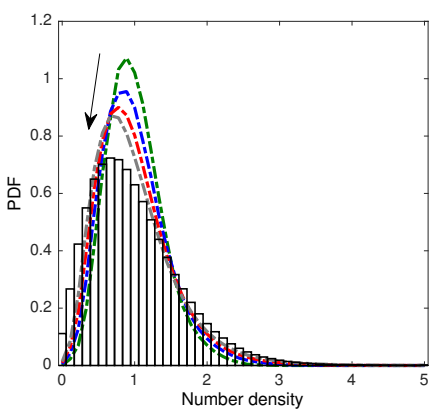

(b) AG

Figure 6: Number density PDF for the Lagrangian (black histogram), AG (dot-dashed lines) and MK (dashed lines) results on $64^{3}$ (green), $128^{3}$ (blue), $256^{3}$ (red) and $512^{3}$ (gray) meshes for $\mathrm{St}_{K}=8$. Arrows indicate increasing mesh sizes for Eulerian simulations.

\subsection{Eulerian velocity}

The second moment of interest is the Eulerian velocity $\boldsymbol{u}$. Here, we directly investigate the distribution function of its component in the x-direction, see Fig. 7. For $\mathrm{St}_{K}=1$, we see again that the MK and AG models are close to the Lagrangian reference, as only a negligible amount of PTC occur and the main part of the particle dynamics is contained into the Eulerian velocity. For $\mathrm{St}_{K}=3$, the AG result matches the Lagrangian one except near zero velocity. For the MK results the velocity in the x-direction is less concentrated near the zero velocity and more spread toward the maximum and minimum values (Fig. 7). The velocity PDF of the MK result is not affected significantly by mesh refinement. For the AG result, the distribution level of the AG velocity approaches the Lagrangian one with mesh refinement, this can be clearly seen near zero-velocity. In fact, in this case the PTC now affect the motion of the disperse phase because $\mathrm{St}_{K}>1$. Finally, for $\mathrm{St}_{K}=8$, the $\mathrm{AG}$ model is again close to the Lagrangian reference, and the velocity distribution is not impacted by mesh refinement. 


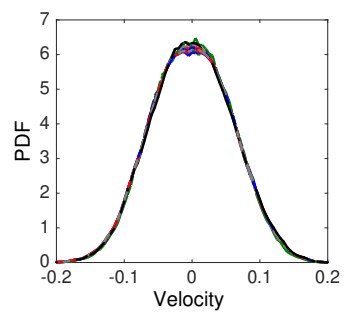

(a) $\mathrm{St}_{K}=1$

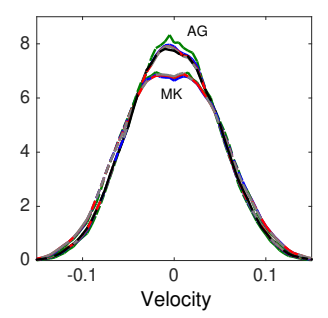

(b) $\mathrm{St}_{K}=3$

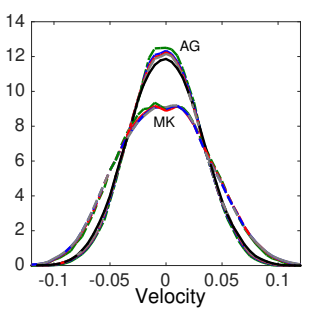

(c) $\mathrm{St}_{K}=8$

Figure 7: PDF of the x-velocity for the Lagrangian (black solid line), AG (dot-dashed line) and MK (dashed line) results on $64^{3}$ (green), $128^{3}$ (blue), $256^{3}$ (red) and $512^{3}$ (grey) meshes for $\mathrm{St}_{K}=1, \mathrm{St}_{K}=3$ and $\mathrm{St}_{K}=8$.

\subsection{Total and internal energies}

As a final validation, we investigate the mean total and internal energies:

$$
\begin{gathered}
\operatorname{MIE}=\frac{\langle n \operatorname{tr}(\boldsymbol{\Sigma})\rangle}{2\langle n\rangle}, \\
\operatorname{MTE}=\frac{\left\langle n\left(\sum_{i} u_{i}^{2}+\operatorname{tr}(\boldsymbol{\Sigma})\right)\right\rangle}{2\langle n\rangle} .
\end{gathered}
$$

The study of the evolution of the mean internal energy (MIE) is essential, since it is through the velocity variance or the internal agitation energy that the AG model is capable of reproducing statistically the PTC. Indeed, at each crossing an amount of the kinetic energy $\frac{n}{2} \sum_{i} u_{i}^{2}$ is transferred into internal energy $\frac{n}{2} \operatorname{tr}(\boldsymbol{\Sigma})$. Then, the MIE is an evaluation of the occurrence of PTC. This mechanism of transfer of energy between the Eulerian velocity and the MIE has been clearly depicted in 4, when looking at the equation of the internal energy:

$$
\partial_{t}(n \operatorname{tr}(\boldsymbol{\Sigma}))+\partial_{\overrightarrow{\boldsymbol{x}}} \cdot(n \operatorname{tr}(\boldsymbol{\Sigma}) \overrightarrow{\boldsymbol{u}})=-\frac{2 n \operatorname{tr}(\boldsymbol{\Sigma})}{\tau_{d}}-n \boldsymbol{\Sigma}: \partial_{\overrightarrow{\boldsymbol{x}}} \overrightarrow{\boldsymbol{u}}
$$

where : is the contraction product of two tensors. In this equation the last term corresponds to the transfer of energy from the Eulerian velocity to the internal energy. Therefore, at crossing events, energy is transferred from the eulerian velocity to the internal energy. 
Next, since the mean total energy (MTE) is the total amount of energy available in the whole domain, the knowledge of both MIE and MTE allows to evaluate the transfer of mesoscopic momentum between the kinetic (correlated motion) and the internal (uncorrelated motion) energies. In theory, this energy transfer can be quantified, see [4].

In Fig. 8 the statistics are plotted for $\mathrm{St}_{K}=1$. In Fig. 8(b) and 8(c) the MTE is underestimated by the MK model, because of the destruction of the kinetic energy during PTC. On the contrary, the AG model performs better and better with mesh refinement. Its MIE is higher compared to the Lagrangian reference but converges toward the right level when the numerical dissipation decreases, thanks to the mesh refinement.

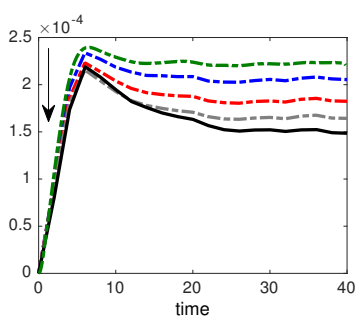

(a) Mean internal energy

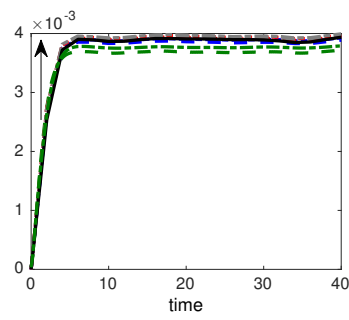

(b) Mean total energy

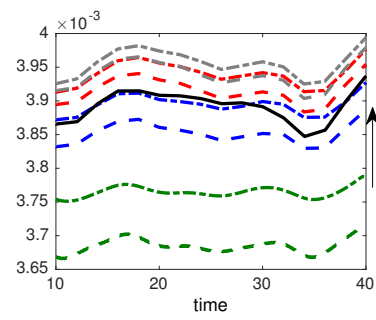

(c) Mean total energy (zoom)

Figure 8: Statistics evolution with time for Lagrangian results (black solid line) and Eulerian results using $64^{3}$ (green), $128^{3}$ (blue), $256^{3}$ (red) and $512^{3}$ (grey) meshes with projection on the same $64^{3}$ mesh for $\mathrm{St}_{K}=1$, for the MK model (dashed lines) and the AG model (dot-dashed lines). Arrows indicate increasing mesh sizes for Eulerian simulations.

In the case of moderately inertial particle $\mathrm{St}_{K}=3$ (Fig. 9), the departure of the MK from the Lagrangian result is obvious. For the AG model, refinement improves the quality of the results for the MTE, but MIE seems to converge to a higher value than the Lagrangian reference.

For an even more inertial disperse phase $\left(\mathrm{St}_{K}=8\right.$, Fig. 10), the AG model has got the right long-time behaviour, but the MTE is now underestimated. 


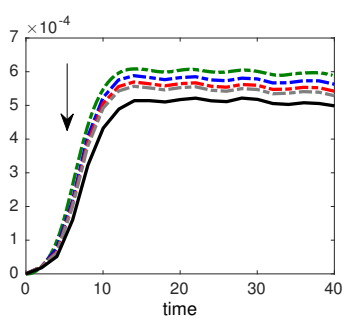

(a) Mean internal energy

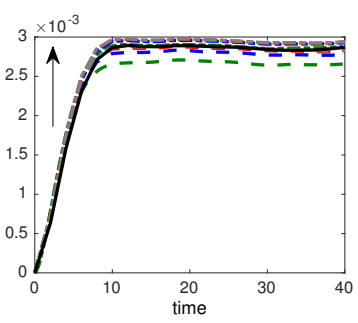

(b) Mean total energy

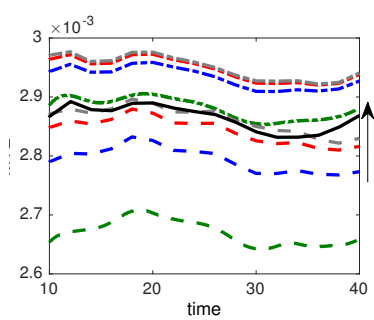

(c) Mean total energy zoom

Figure 9: Statistics evolution with time for Lagrangian results (black solid line) and Eulerian results using $64^{3}$ (green), $128^{3}$ (blue), $256^{3}$ (red) and $512^{3}$ (grey) meshes with projection on the same $64^{3}$ mesh for $\mathrm{St}_{K}=3$, for the MK model (dashed lines) and the AG model (dot-dashed lines). Arrows indicate increasing mesh sizes for Eulerian simulations.

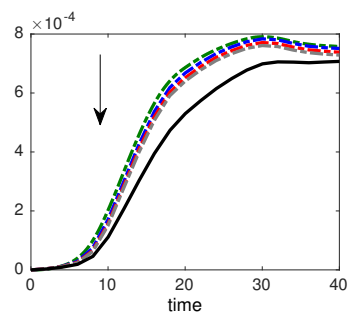

(a) Mean internal energy

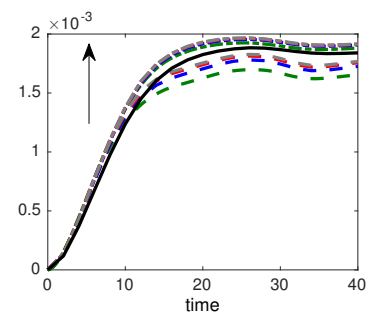

(b) Mean total energy

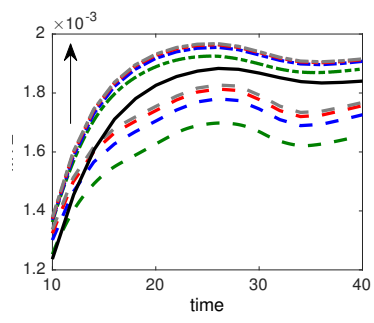

(c) Mean total energy zoom

Figure 10: Statistics evolution with time for Lagrangian results (black solid line) and Eulerian results using $64^{3}$ (green), $128^{3}$ (blue), $256^{3}$ (red) and $512^{3}$ (grey) meshes with projection on the same $64^{3}$ mesh for $\mathrm{St}_{K}=8$, for the MK model (dashed lines) and the AG model (dotdashed lines). Arrows indicate increasing mesh sizes for Eulerian simulations.

\subsubsection{PDF of the trace of the pressure tensor}

To accurately evaluate the degree of PTC captured by the AG model, the distribution functions of the trace of the pressure tensor for the Lagrangian and AG simulations on different meshes are plotted in Fig. 11. For $\mathrm{St}_{K}=1$, the distribution functions of the AG results converge to the Lagrangian one when refining the mesh. Most of the particle eulerian field has zero or very small pressure, since, as explained previously, the probability of having local Stokes numbers higher than the critical one is non-negligible.

For moderately inertial particles, $\mathrm{St}_{K}=3$, the form of the PDF of the trace 
of the pressure tensor of the AG result matches the Lagrangian one at high pressures. However, the number of low-pressure particles is underestimated by the AG results, especially on the coarsest mesh. When refining the mesh, the difference between the AG and Lagrangian distribution functions of the internal energy decreases. Actually, the internal energy includes two contributions:

- one is purely related to the model and is the reason why AG is able to capture PTC;

- an other one is due to numerical error; because the numerical scheme is "mixing" states of different velocities and energies, it necessarily leads to the creation of "numerical" PTC, which contribute to the overall internal energy.

This explains the convergence of the PDF of the internal energy using the AG model when refining the mesh, since by doing so, the second contribution is decreased and the physical behaviour becomes predominant; whereas for the coarsest mesh, the dominant effect is the numerical diffusion.

For the highest Stokes number, $\mathrm{St}_{K}=8$, the PDF of the trace of the pressure tensor for the AG result is still close to the Lagrangian one.

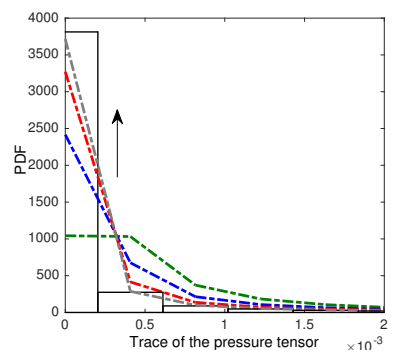

(a) $\mathrm{St}_{K}=1$

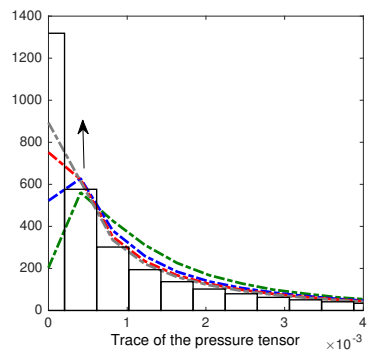

(b) $\mathrm{St}_{K}=3$

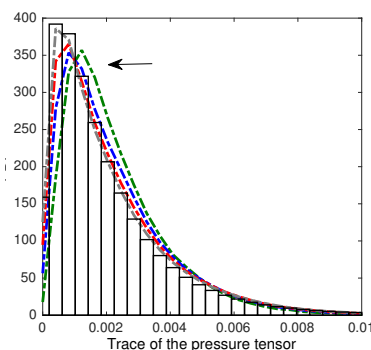

(c) $\mathrm{St}_{K}=8$

Figure 11: PDF of the pressure for the Lagrangian (black histogram) and AG results on $64^{3}$ (green), $128^{3}$ (blue), $256^{3}$ (red) and $512^{3}$ (grey) meshes for $\mathrm{St}_{K}=1, \mathrm{St}_{K}=3$ and $\mathrm{St}_{K}=8$. Arrows indicate increasing mesh sizes for Eulerian simulations. 


\subsection{Statistical quantities evolution with Stokes number}

Finally, to summarize the ability of the AG model to reproduce the statistics of the turbulent particle-laden flow, we look at the results at a final time that depends on the Stokes number in order to reach a stationary state. This final time $t_{f}$ is equal to the maximum between $4 \tau_{d}$ and the eddy turn over time of the turbulence.

The results of the AG model are plotted in Fig. 12 for two meshes $\left(128^{3}\right.$ and $512^{3}$ ), for the segregation, the total and the internal energy. Two vertical lines also indicate the unity Stokes numbers based on the Lagrangian integral time scale of the fluid $\left(\mathrm{St}_{L}\right)$ and the Eulerian time scale of the turbulence $\mathrm{St}_{E}=$ $\tau_{p} / \tau_{\text {eddy }}$. Overall, it clearly appears that the AG model is suitable as long as $\mathrm{St}_{L}=\tau_{p} / \tau_{L}$ is below one, and still gives reasonable results as long as $\mathrm{St}_{E}$ is below one. Total energy is still always well predicted, and the energy partition between total and internal energy is well captured compared to the Lagrangian reference, as shown in Fig. 13 .

\section{Conclusion}

In this paper, the Anistropic Gaussian model, designed to capture Particle Trajectory Crossings, has been evaluated on a 3D Forced Homogeneous Isotropic Turbulence loaded with a dilute disperse phase. The AG results are compared with a statistically-converged Lagrangian reference and with the MonoKinetic closure, a simpler closure not capable of dealing with PTC. From the qualitative and statistical results presented in this article, several conclusions can be drawn.

First, for particles having small inertia $\mathrm{St}_{K}<1$, both Eulerian models (MK and $A G$ ) lead to accurate results. The qualitative and quantitative results highlight the inference that the MK and the AG reproduce the right physics in this case, in comparison with the Lagrangian results. The MK model has the advantage of being less expensive than the AG model since in $3 \mathrm{D}$, the number of equations to be solved is only 4, versus 10 equations for the AG model. Thus, MK is preferred to AG for the simulation of a disperse phase having a Stokes 


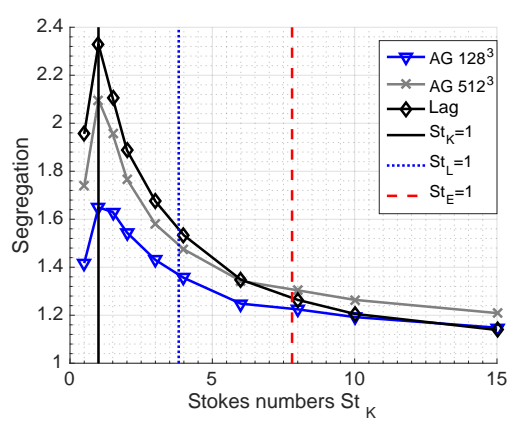

(a)

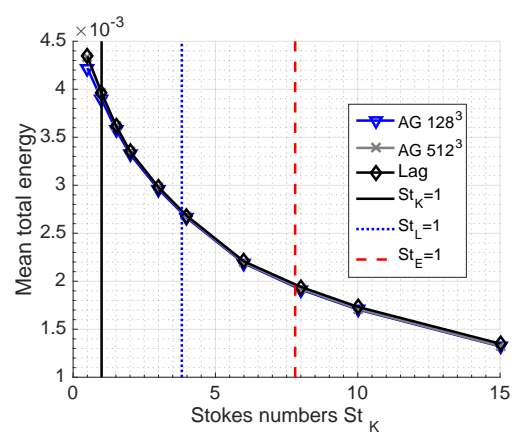

(b)

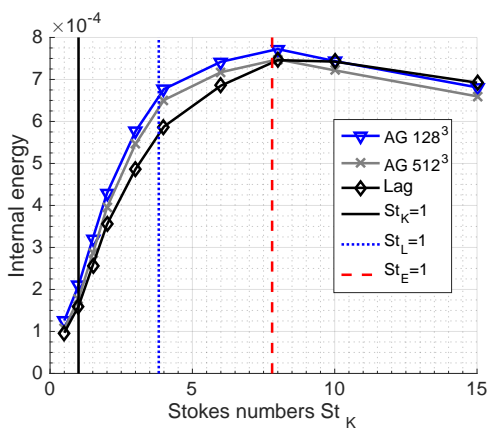

(c)

Figure 12: Segregation (a), Total energy (b) and internal energy (c) as a function of the Stokes number for Lagrangian result and Anisotropic Gaussian results on the $128^{3}$ and $512^{3}$ meshes with projection on the same $64^{3}$ mesh at $t_{f}$. The vertical lines are: $S t_{K}=1$ in black, $S t_{L}=1$ in blue and $S t_{E}=1$ in red.

number based on the Kolmogorov scale less than 1. It is important to note that this model can be more accurate whenever solved with adapted numerical methods such as a Finite Volume Kinetic Scheme or a realizability preserving RKDG [40, 19. However, one should keep in mind the fact that the AG model has the advantage of capturing PTC that can locally occur when the local Stokes number overcomes the critical one.

Second, for a moderately inertial to inertial disperse phase, the AG model gives the right qualitative behavior, whereas the MK model ceases to repro- 


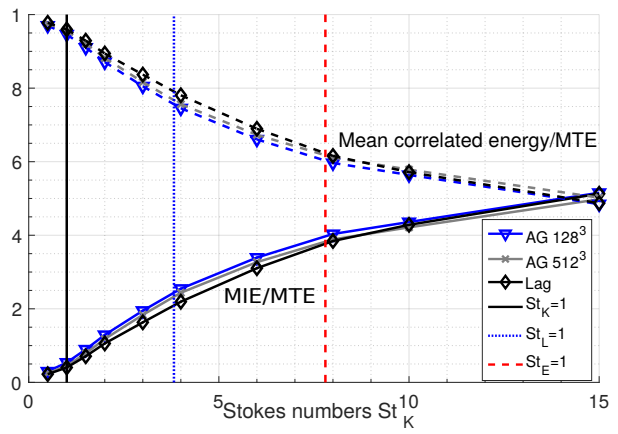

Figure 13: Energy partition as a function of the Stokes number for Lagrangian result and Anisotropic Gaussian results on the $128^{3}$ and $512^{3}$ meshes with projection on the same $64^{3}$ mesh at $t_{f}$. The vertical lines are: $S t_{K}=1$ in black, $S t_{L}=1$ in red and $S t_{E}=1$ in blue.

duce the right dynamics of the disperse phase for Stokes numbers $S t_{K}>1$. In addition, whenever the particle relaxation time is in the range between the Kolmogorov time scale and the integral time scale characterizing the large eddies, the temporal evolution of the statistical mean variables is captured precisely with the AG model.

Finally, for very inertial particles, $S t_{L} \geq 1$, the AG model gives much better results than the MK model, but starts to depart from the Lagrangian reference, if we look at the statistics of the number density field, even if the Eulerian velocity and the energies are relatively well captured. This is especially true in the long time behaviour. The limits of the AG model can be explained by the fact that it solves for a finite number of data of the statistics of the PTC: it captures only the local effects of the trajectory crossing. Yet, when the Stokes number grows, particles start to cross at a much larger scale and the AG model ceases to be relevant.

Based on these different results, the domain of relevance of the AG model extends from the unity Stokes number based on the Kolmogorov scale to the unity Stokes number based on the integral scale. For higher Stokes number for which AG starts to depart from the Lagrangian reference because of large-scale crossings, our model can be embedded within a Large Eddy Simulation context, 
for which these PTC will contribute to an additional subgrid scale pressure [4].

\section{Appendix A. Numerical convergence of the Eulerian simulations}

In order to compare the Eulerian models in the context of a refinement process, the results for MK and AG have been presented on the $64^{3}, 128^{3}$, $256^{3}$ and $512^{3}$ meshes in the result section. Hence, we aim at separating the modeling influence from the numerical contributions on the capacity of our strategy in predicting the proper physical behavior of the flow. Ideally, we would be interested in the comparison of the models at numerical convergence, which is hard to reach.

In this Appendix, we wish to go one step further in the convergence analysis, and look at a qualitative assessment of the convergence of the different considered fields, as well as at a grid convergence index and regression tool in order to estimate a converged solution.

\section{Appendix A.1. Qualitative analysis of the Eulerian field convergence}

First, we focus on the case St $=0.5$ at time $t=40$, since the flow is really segregated and it is one of the most difficult one in terms of convergence, and consider various result fields on $64^{3}, 128^{3}, 256^{3}$ and $512^{3}$ meshes. The study is only shown for the MK results, since at this Stokes number the results for both Eulerian models are nearly the same. This comparison is performed on the 2-D diagonal slice shown in Fig. A.14. From Fig. 15(a), we observe that the number

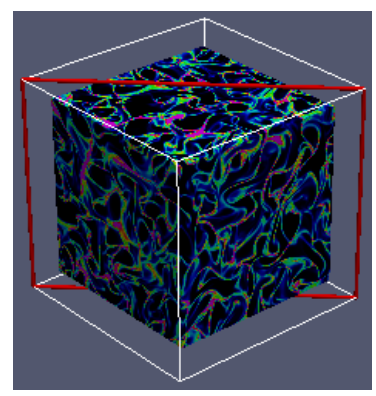

Figure A.14: The diagonal slice plan shown in red 
density field using MK is close to the Lagrangian one, even if some small details are slightly different. The result on the $512^{3}$ grid captures the right structures, the correct vacuum zones and even the thin high concentration filaments and very small difference appears between the $256^{3}$ and $512^{3}$ meshes.

For the velocity, the results are not highly affected by mesh refinement, as shown in Fig. 15(c), For the coarsest mesh the right motion is already captured, except near the vacuum regions. Same conclusions can be drawn from the energy fields.

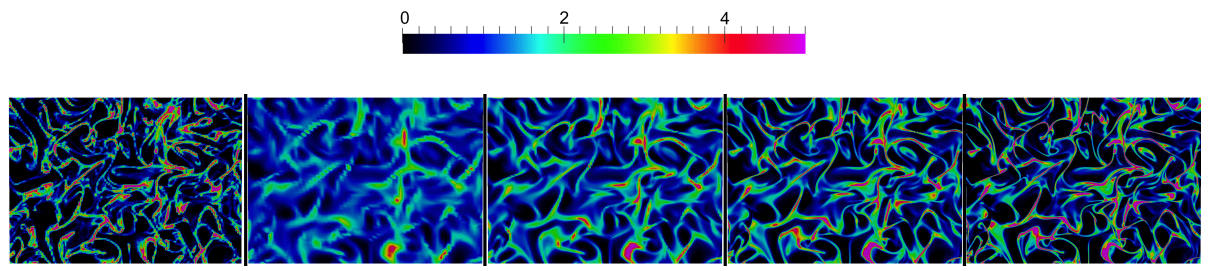

(a) Number density

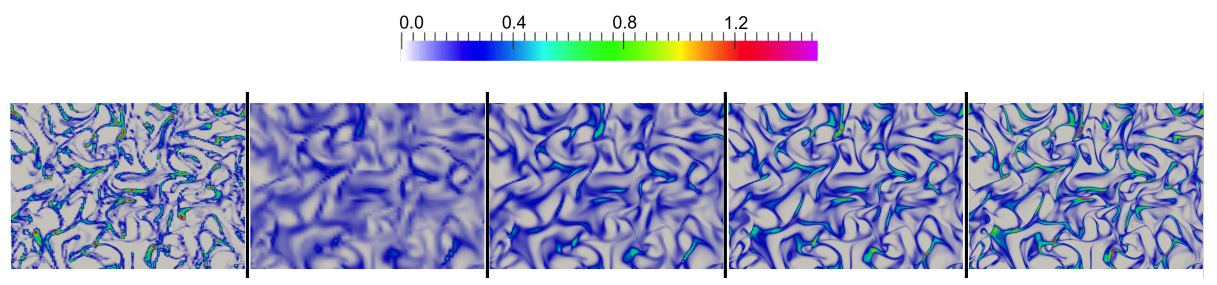

(b) Momentum

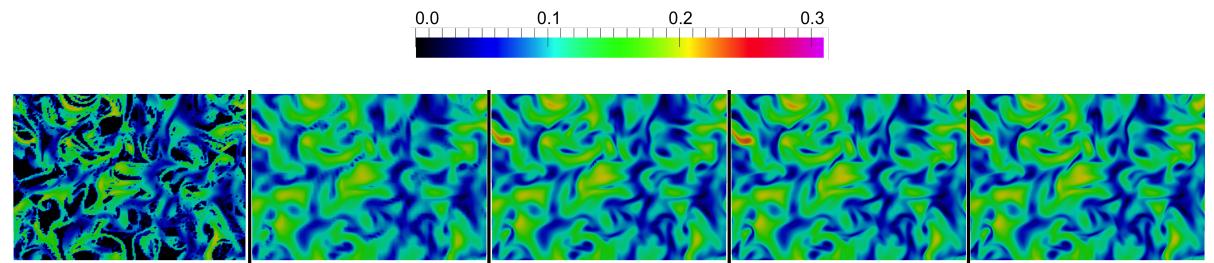

(c) Velocity

Figure A.15: From left to right: Lagrangian, MK on $64^{3}, 128^{3}, 256^{3}$ and $512^{3}$ meshes at $t=40$ for $\mathrm{St}=0.5$

So far the qualitative assessment of the fields convergence seems achieved, but a further inquiry is necessary in order to reach quantitative conclusions. 
Appendix A.2. Convergence rate of the statistics for the AG model

We propose to analyze further the convergence of the simulations relying on the AG model. To do so, we use the Grid Convergence Index [42], which goal is to estimate the convergence rate as well as an estimate of the converged solution, based on three results with different meshes. If $\varepsilon_{128}, \varepsilon_{256}$ and $\varepsilon_{512}$ are three scalar quantities of interest post-processed from the $128^{3}, 256^{3}$ and $512^{3}$ meshes and if we suppose that we have reached the asymptotic convergence regime of the numerical scheme, we may postulate that the variable $\varepsilon$ evolves with the mesh refinement as:

$$
\varepsilon_{k}-\varepsilon^{*}=C\left(h_{k}\right)^{p}, \quad k=128,256,512,
$$

where $\varepsilon^{*}$ is the theoretical value of the quantity $\varepsilon$ at convergence, $C$ is a constant, $h_{k}$ is the characteristic size of mesh $k$, and $p$ is an estimate of the order of convergence. If we are in the asymptotic regime these two constants, $C, p$ are fixed and from the three equations A.1 we can obtain the values of the constants as well as an estimate of the scalar field at convergence:

$$
p=-\frac{1}{\log (2)} \log \frac{\left|\varepsilon_{512}-\varepsilon_{256}\right|}{\left|\varepsilon_{256}-\varepsilon_{128}\right|}, \quad C=\frac{\varepsilon_{512}-\varepsilon_{256}}{h_{512}^{p}-h_{256}^{p}}, \quad \varepsilon^{*}=\frac{\varepsilon_{512}-0.5^{p} \varepsilon_{256}}{1-0.5^{p}} .
$$

We thus get an estimate of the finest result in the asymptotic regime using:

$$
\varphi=\left|\frac{\varepsilon^{*}-\varepsilon_{512}}{\varepsilon^{*}}\right| .
$$

Such an analysis relies heavily on the fact that we have reached the asymptotic regime for the numerical scheme. If not, it could lead to inaccurate orders and meaningless converged solutions.

In the following, this analysis is performed for the evolution of the mean total energy plotted and of the segregation in Figs. 8(b), 9(b), 10(b) and 3 The evolution with time of the estimated values at convergence of the MTE for

\footnotetext{
${ }^{2}$ For large enough discretization steps, we may be in a non-asymptotic regime and the ordre of the method can change with the step size as analyzed in [43].
} 
Stokes numbers 1, 3 and 8 is plotted in Fig. A.16. The same has been done for the segregation, see Fig. A.17.

Looking at the MTE, the asymptotic convergence regime seems to be reached, with a convergence rate point-by-point in time $p \in[1,2]$ and most of the values greater than 1.5. Also, the convergence estimate is very close to the reference Lagrangian values.

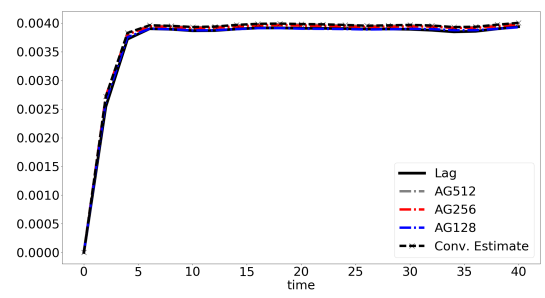

(a) $\mathrm{St}_{K}=1$

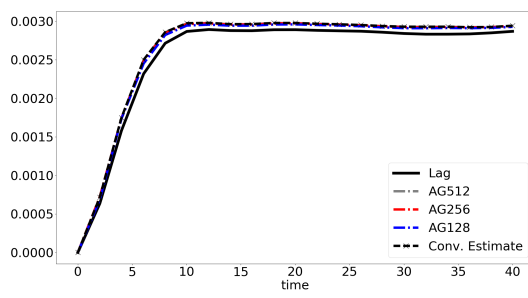

(b) $\mathrm{St}_{K}=3$

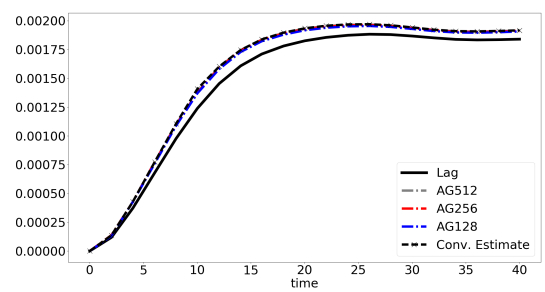

(c) $\mathrm{St}_{K}=8$

Figure A.16: Time evolution of the MTE for the Lagrangian result (black solid line), the AG results (dot-dashed lines) with $128^{3}$ (grey), $256^{3}$ (red) and $512^{3}$ (blue) cells, and the estimate of the segregation at convergence (dashed line with crosses), for three Stokes number.

However, when looking at the segregation, the computed orders of convergence are around 0.12 at $\mathrm{St}=1,0.5$ at $\mathrm{St}=3$ and 0.25 at $\mathrm{St}=8$, which indicates that we are not yet in the convergence regime, even questioning if we can reach such a regime using the segregation scalar field in such a case. In these conditions, it is obvious that the asymptotic regime has not been reached. This is even clearer when looking at the maximum of the extrapolated error A.3 in time, see table A.3. When the convergence error for the mean total energy is globally lower than two percent, it is higher than $10 \%$ when looking at the segregation of the disperse field and reacher $60 \%$ for the most segregated 


\begin{tabular}{|l|c|c|c|}
\hline Stokes Number & 1 & 3 & 8 \\
\hline Segregation & $59.85 \%$ & $10.58 \%$ & $21.22 \%$ \\
\hline Mean Total Energy & $0.28 \%$ & $0.16 \%$ & $2.09 \%$ \\
\hline
\end{tabular}

Table A.3: Maximum of the extrapolated error $(t \in[0,40])$ for segregation and MTE.

case. At this point, it has to be kept in mind that, even if segregation is a classical metric used in the literature to quantify number density fields, it is still a metric that strongly magnifies errors on depletion zones and fine regions of stiff accumulation because of its quadratic nature, and such regions are highly affected by numerical errors, such as the extra dissipation coming from the limiting strategies necessary to ensure stability and robustness of our simulations. Looking at the evolution of the PDF in number density in Fig. 4.1.3, the NDF is well predicted, showing that segregation is not necessarily a metric for which we can analyze mesh convergence in such highly segregated flows.

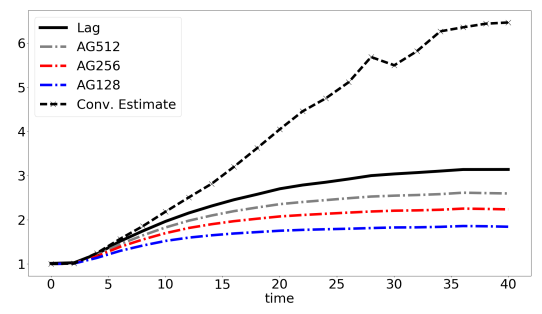

(a) $\mathrm{St}_{K}=1$

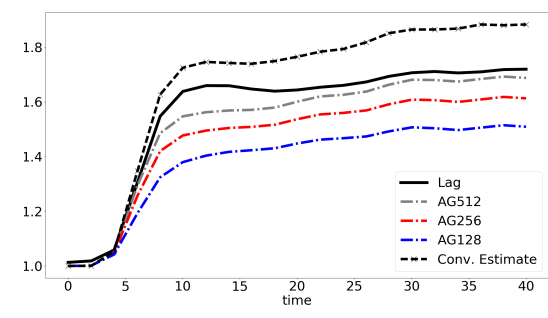

(b) $\mathrm{St}_{K}=3$

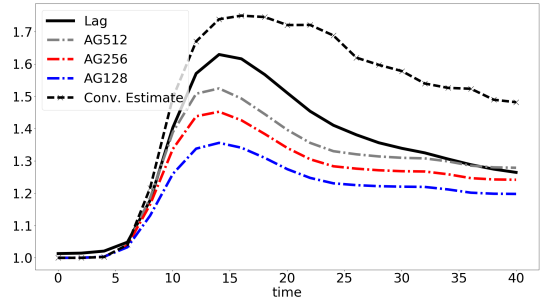

(c) $\mathrm{St}_{K}=8$

Figure A.17: Time evolution of the segregation for the Lagrangian result (black solid line), the AG results (dot-dashed lines) with $128^{3}$ (grey), $256^{3}$ (red) and $512^{3}$ (blue) cells, and the estimate of the segregation at convergence (dashed line with crosses), for three Stokes number. 


\section{Appendix B. A Realizable Second-Order Dedicated Finite Volume Scheme}

Here, the realizable second-order MUSCL/HLL scheme [1] discussed in Sec. 2.4 is detailed. It is based on the MUSCL strategy [44, using a linear conservative reconstruction of the primitive variables, a realizable HLL scheme, and a Strong-Stability-Preserving Runge-Kutta method. Multi-dimensional problems are solved by a dimensional splitting strategy, so we focus on $\mathrm{x}$-direction numerical building block. For more details, one may refer to the work of [1].

Appendix B.1. Realizable and conservative reconstruction

Realizability constraints on the moment set are expressed more easily on the primitive variables. Therefore, we apply the linear reconstruction on these variables, and we impose moment conservation in each cell to ensure realizability of the numerical scheme [1].

It starts by the evaluation of local slopes by means of the neighboring mean values $\overline{\mathcal{V}}$ :

$$
\widetilde{\mathcal{V}}_{i}(x)=\overline{\mathcal{V}}_{i}+\mathcal{D} \mathcal{V}_{i}\left(x-x_{i}\right)
$$

Contrary to the classical MUSCL reconstruction [45], the reconstruction of the

primitive variables $\mathcal{V}=(n, \boldsymbol{u}, \boldsymbol{\Sigma})^{T}$ needs to be corrected in order to ensure the conservation of the conserved variables $\mathcal{W}=(n, n \boldsymbol{u}, n \mathbf{E})^{T}$ :

$$
\frac{1}{\Delta x} \int_{x_{i-1 / 2}}^{x_{i+1 / 2}} \widetilde{\mathcal{W}}_{i}(x) \mathrm{d} x=\overline{\mathcal{W}}_{i}
$$

where index $i$ refers to the cell $i$. These latest constraints imply the following corrected primitive mean values:

$$
\begin{aligned}
\bar{n}_{i} & =n_{i}, \quad \overline{\boldsymbol{u}}_{i}=\boldsymbol{u}_{i}-\frac{D_{n, i} \mathbf{D}_{\boldsymbol{u}, i}}{n_{i}} \frac{\Delta x^{2}}{12} \\
\overline{\boldsymbol{\Sigma}}_{i} & =\boldsymbol{\Sigma}_{i}-\frac{\Delta x^{2}}{12} \alpha \mathbf{D}_{\boldsymbol{u}, i}^{2}-\frac{\Delta x^{2}}{12} \frac{D_{n, i}}{n_{i}} \mathbf{D}_{\boldsymbol{\Sigma}, \boldsymbol{i}}
\end{aligned}
$$


where:

$$
\mathbf{D}_{\boldsymbol{u}, i}^{2}=\left(\begin{array}{ccc}
D_{u_{i}}^{2} & D_{u_{i}} D_{v_{i}} & D_{u_{i}} D_{w_{i}} \\
D_{u_{i}} D_{v_{i}} & D_{v_{i}}^{2} & D_{v_{i}} D_{w_{i}} \\
D_{u_{i}} D_{w_{i}} & D_{v_{i}} D_{w_{i}} & D_{w_{i}}^{2}
\end{array}\right), \mathbf{D}_{\boldsymbol{\Sigma}, i}=\left(\begin{array}{ccc}
D_{\sigma_{11}, i} & D_{\sigma_{12}, i} & D_{\sigma_{13}, i} \\
D_{\sigma_{12}, i} & D_{\sigma_{22}, i} & D_{\sigma_{32}, i} \\
D_{\sigma_{13}, i} & D_{\sigma_{23}, i} & D_{\sigma_{33}, i}
\end{array}\right)
$$

and $\alpha=1+\frac{\Delta x^{2}}{12} \frac{D_{n, i}^{2}}{n_{i}^{2}}$.

\section{Appendix B.2. Slope Limitation}

Eq. B.5 shows a non-linear coupling between the reconstruction of each primitive variables. Therefore, ensuring realizability constraint on a specific variable may affect the other variables. In the following, we propose a sequential strategy that limits the need of a non-linear optimization to find the best choice:

1. First the density field $n_{i}$ is limited using a classical minmod limiter that ensures its positivity.

2. Next, the slopes for velocity are also limited to ensure the constraint of positive internal energy. This limitation reads:

$$
\begin{aligned}
D_{\boldsymbol{u}, i} & =\frac{1}{2}\left(\operatorname{sign}\left(\boldsymbol{u}_{i+1}-\boldsymbol{u}_{i}\right)+\operatorname{sign}\left(\boldsymbol{u}_{i}-\boldsymbol{u}_{i-1}\right)\right) \\
& \min \left(\frac{\left|\boldsymbol{u}_{i+1}-\boldsymbol{u}_{i}\right|}{\Delta x\left(1-\frac{D_{n_{i}}}{n_{i}} \frac{\Delta x}{6}\right)}, \frac{\left|\boldsymbol{u}_{i}-\boldsymbol{u}_{i-1}\right|}{\Delta x\left(1+\frac{D_{n_{i}}}{n_{i}} \frac{\Delta x}{6}\right)}, \mathbf{D}_{\boldsymbol{u}, i}^{\max , \boldsymbol{\Sigma}}, \frac{1}{\Delta t}\right),
\end{aligned}
$$

where:

$$
\mathbf{D}_{\boldsymbol{u}, i}^{\max , \boldsymbol{\Sigma}}=\sqrt{\frac{\operatorname{diag}(\boldsymbol{\Sigma})}{\frac{\Delta x^{2}}{12}\left(1+\frac{\Delta x^{2}}{12} \frac{D_{n_{i}}^{2}}{n_{i}^{2}}\right)}}
$$

To ensure the positivity of the determinant of the covariance matrix $\boldsymbol{\Sigma}$, an additional procedure is used (not detailed here), in the same way as in [1] for the 2D case: if the computed slopes generate a negative determinant, an additional limitation is applied on the slopes, knowing that for zero slopes, realizability is ensured. 
3. Finally, the components of the covariance matrix $\Sigma$ are limited in order to ensure the preservation of the positivity of its diagonal terms:

$$
\begin{aligned}
\operatorname{diag}\left(\mathbf{D}_{\boldsymbol{\Sigma}, i}\right)= & \frac{1}{2}\left[\operatorname{sign}\left(\operatorname{diag}\left(\boldsymbol{\Sigma}_{i+1}^{*}\right)-\operatorname{diag}\left(\boldsymbol{\Sigma}_{i}^{*}\right)\right)+\operatorname{sign}\left(\operatorname{diag}\left(\boldsymbol{\Sigma}_{i}^{*}\right)-\operatorname{diag}\left(\boldsymbol{\Sigma}_{i-1}^{*}\right)\right)\right] \\
& \times \min \left(\frac{\left|\operatorname{diag}\left(\boldsymbol{\Sigma}_{i+1}^{*}\right)-\operatorname{diag}\left(\boldsymbol{\Sigma}_{i}^{*}\right)\right|}{\Delta x\left(1-\frac{D_{n_{i}}}{\rho_{j}} \frac{\Delta x}{6}\right)}, \frac{\left|\operatorname{diag}\left(\boldsymbol{\Sigma}_{i}^{*}\right)-\operatorname{diag}\left(\boldsymbol{\Sigma}_{i-1}^{*}\right)\right|}{\Delta x\left(1+\frac{D_{n_{i}}}{n_{i}} \frac{\Delta x}{6}\right)}\right)
\end{aligned}
$$

where $\boldsymbol{\Sigma}^{*}=\boldsymbol{\Sigma}_{j}-\frac{\Delta x^{2}}{12} \alpha \mathbf{D}_{\boldsymbol{u}}^{2}$. To ensure the positivity of the determinant of $\boldsymbol{\Sigma}$, a non-linear optimisation is still required, as in $[1]$.

\section{Appendix B.3. Flux evaluation}

The numerical flux evaluation is performed by means of a classical HLL approximate Riemann solver [46]:

$$
\begin{aligned}
\mathcal{F}^{H L L}\left(\mathcal{W}_{L}, \mathcal{W}_{R}\right)=\frac{1}{2}\left(\mathcal{F}\left(\mathcal{W}_{L}\right)+\mathcal{F}\left(\mathcal{W}_{R}\right)\right) & -\frac{1}{2}\left|\lambda_{\min }\right|\left(\mathcal{W}^{*}-\mathcal{W}_{L}\right) \\
& -\frac{1}{2}\left|\lambda_{\max }\right|\left(\mathcal{W}_{R}-\mathcal{W}^{*}\right)
\end{aligned}
$$

where the intermediate state $\mathcal{W}^{*}$ is

$$
\mathcal{W}^{*}=\frac{\lambda_{\min } \mathcal{W}_{L}-\lambda_{\max } \mathcal{W}_{R}}{\lambda_{\min }-\lambda_{\max }}-\frac{\mathcal{F}\left(\mathcal{W}_{L}\right)-\mathcal{F}\left(\mathcal{W}_{R}\right)}{\lambda_{\min }-\lambda_{\max }},
$$

$R$ and $L$ stand respectively for the right and left side of the interface and $\lambda_{\min }$ and $\lambda_{\max }$ are respectively the slowest and fastest characteristic waves of the states on the left and right side of the interface.

This standard flux can be shown to be realizability-preserving in the sense that, if $\mathcal{S}$ denotes the convex realizability constraints, then $\mathcal{W}_{i-1}^{n}, \mathcal{W}_{i}^{n}, \mathcal{W}_{i+1}^{n} \in$ $\mathcal{S}$ at time step $n$ implies that the explicit first order Euler update

$$
\mathcal{W}_{i}^{n+1}=\mathcal{W}_{i}^{n}-\frac{\Delta t}{\Delta x}\left(\mathcal{F}^{H L L}\left(\mathcal{W}_{i}^{n}, \mathcal{W}_{i+1}^{n}\right)-\mathcal{F}^{H L L}\left(\mathcal{W}_{i-1}^{n}, \mathcal{W}_{i}^{n}\right)\right)
$$

also belongs to $\mathcal{S}$ under the classical CFL condition on the ratio $\frac{\Delta t}{\Delta x}$ for first order finite volume schemes 47 .

Then, by means of a strong stability preserving (SSP) 2-step Runge-Kutta method, we reach second order in time while preserving the realizability of the update. 


\section{Acknowledgements}

The authors acknowledge SAFRAN-SNECMA for the financial support of the $\mathrm{PhD}$ of M. Sabat. This work was granted access to the HPC resources of CINES under the allocation x2016026172 made by GENCI (Grand Equipement National de Calcul Intensif). The authors also acknowledge IDRIS for the computation resources on Ada. The help of M. Boileau, J.C. Brändle de Motta, O. Thomine and L. Freret on Asphodele/Muses3D codes is gratefully acknowledged.

\section{References}

[1] A. Vié, F. Doisneau, M. Massot, On the Anisotropic Gaussian closure for the prediction of inertial-particle laden flows, Comm. in Comp. Physics 17 (1) (2015) 1-46.

[2] S. Subramaniam, Lagrangian-Eulerian methods for multiphase flows, Progress in Energy and Combustion Science 231 (2-3) (2013) 215-245.

[3] J. Capecelatro, O. Desjardins, R. O. Fox, Strongly coupled fluid-particle flows in vertical channels. II. Turbulence modeling, Physics of Fluids 28 (3) (2016) $1-23$.

[4] P. Février, O. Simonin, K. D. Squires, Partitioning of particle velocities in gas-solid turbulent flow into a continuous field and a spatially uncorrelated random distribution: theoretical formalism and numerical study, J. Fluid Mech. 533 (2005) 1-46.

[5] A. Kaufmann, M. Moreau, O. Simonin, J. Helie, Comparison between Lagrangian and mesoscopic Eulerian modelling approaches for inertial particles suspended in decaying isotropic turbulence, Journal of Computational Physics 227 (2008) 6448-6472.

[6] E. Masi, Simonin, Algebraic-Closure-Based Moment Method for unsteady Eulerian simulations of non-isothermal particle-laden turbulent flows at 
moderate Stokes numbers in dilute regime, Flow Turbulence and Combustion 92 (1-2) (2014) 121-145.

[7] E. Masi, O. Simonin, E. Riber, P. Sierra, L. Gicquel, Development of an algebraic-closure-based moment method for unsteady Eulerian simulations of particle-laden turbulent flows in very dilute regime, International Journal of Multiphase Flow 58 (2014) 257-278.

[8] N. Le Lostec, R. O. Fox, O. Simonin, P. Villedieu, Numerical description of dilute particle-laden flows by a quadrature-based moment method, Proceedings of the Summer Program 2018. Center for Turbulence Research, Stanford (2008) 209-221.

[9] M. Sakiz, O. Simonin, Numerical experiments and modelling of nonequilibrium effects in dilute granular flows, in: Proceeding of 21st International Symposium on Rarefied Gas Dynamics, Vol. 1, Cepadues Editions, Marseille, 1998, pp. 287-294.

[10] C. P. T. Groth, J. G. McDonald, Towards physically-realizable and hyperbolic moment closures for kinetic theory, Continuum Mechanics and Thermodynamics 21 (6) (2009) 467-493.

[11] C. K. S. Lam, C. P. T. Groth, Numerical prediction of three-dimensional non- equilibrium gaseous flows using the gaussian moment closure, AIAA paper 2011-3401.

[12] F. Laurent, A. Vié, C. Chalons, R. Fox, M. Massot, A hierarchy of Eulerian models for trajectory crossing in particle-laden turbulent flows over a wide range of stokes numbers, in: Annual Research Briefs 2012, Center for Turbulence Research, Stanford University, USA, 2012, pp. 193-204.

[13] J. G. McDonald, C. P. T. Groth, Towards realizable hyperbolic moment closures for viscous heat-conducting gas flows based on a maximum-entropy distribution, Continuum Mechanics and Thermodynamics 25 (2013) 573603. 
[14] C. Chalons, R. Fox, F. Laurent, M. Massot, A. Vié, Multivariate gaussian extended quadrature method of moments for turbulent disperse multiphase flow, SIAM Journal on Multiscale Modeling and Simulation 15 (4) (2017) $1553-1583$.

[15] B. Kong, R. Fox, H. Feng, J. Capecelatro, R. Patel, O. Desjardins, Eulereuler anisotropic gaussian mesoscale simulation of homogeneous clusterinduced gas-particle turbulence, AIChE Journal 63 (2017) 2630-2643.

[16] A. Vié, E. Masi, O. Simonin, M. Massot, On the direct numerical simulation of moderate-stokes-number turbulent particulate flows using AlgebraicClosure-Based and Kinetic-Based Moment Methods, in: Proceedings of the summer program 2012, Center for Turbulence Research, Stanford University, 2012, pp. 1-10.

[17] C. Chalons, D. Kah, M. Massot, Beyond pressureless gas dynamics: quadrature-based velocity moment models, Communication in Mathematical Sciences 10 (4) (2012) 1241-1272.

[18] E. Toro, Riemann Solvers and Numerical Methods for Fluid Dynamics: A Practical introduction, 3rd Edition, Springer, 2009.

[19] A. Larat, M. Massot, A. Vié, A stable, robust and high order accurate numerical method for Eulerian simulation of spray and particle transport on unstructured meshes, in: Annual Research Briefs 2012, Center for Turbulence Research, Stanford University, USA, 2012, pp. 205-216.

[20] S. de Chaisemartin, L. Fréret, D. Kah, F. Laurent, R. Fox, J. Reveillon, M. Massot, Eulerian models for turbulent spray combustion with polydispersity and droplet crossing, Comptes Rendus Mécanique 337 (2009) 438-448, special Issue 'Combustion for Aerospace Propulsion'.

[21] M. R. Maxey, J. J. Riley, Equation of motion for a small rigid sphere in a nonuniform flow, Physics of Fluids 26 (4) (1983) 883-889. 
[22] C. Levermore, Moment Closure Hierarchies for Kinetic Theories, Journal of Statistical Physics 83 (5-6) (1996) 1021-1065.

[23] F. Bouchut, On zero pressure gas dynamics, in: Advances in kinetic theory and computing, Vol. 22 of Ser. Adv. Math. Appl. Sci., World Sci. Publ., River Edge, NJ, 1994, pp. 171-190.

[24] F. Marble, Dynamics of dusty gases, Ann. Rev. Fluid Mech. 2 (1970) 397446.

[25] Y. Brenier, E. Grenier, Sticky particles and scalar conservation laws, SIAM Journal of Numerical Analysis 35 (1998) 2317-2328.

[26] C. Levermore, W. Morokoff, The Gaussian moment closure for gas dynamics, SIAM Journal on Applied Mathematics 59 (1) (1998) 72-96.

[27] L. Holway Jr., New statistical models for kinetic theory: methods of construction, Physics of Fluids 9 (9) (1966) 1658-1673.

[28] P. Andries, P. Le Tallec, J. Perlat, B. Perthame, The Gaussian-BGK model of Boltzmann equation with small Prandtl number, Eur. J. of Mech. B Fluids 19 (2000) 813-930.

[29] S. Brown, P. Roe, C. Groth, Numerical solution of a 10-moment model for non-equilibrium gas dynamics, in: 12th AIAA Computational Fluid Dynamics Conference, Fluid Dynamics, 1995.

[30] C. Berthon, Robustness of MUSCL schemes for 2D unstructured meshes, Journal of Computational Physics 218 (2) (2006) 495-509.

[31] C. Berthon, Numerical approximations of the 10-moment Gaussian closure, Mathematical of Computation 75 (256) (2006) 1809-1831.

[32] F. Bouchut, S. Jin, X. Li, Numerical approximations of pressureless and isothermal gas dynamics, SIAM Journal on Numerical Analysis 41 (2003) $135-158$. 
[33] J. Reveillon, F. Demoulin, Effects of the preferential segregation of droplets on evaporation and turbulent mixing, Journal of Fluid Mechanics 583 (2007) 273-302.

[34] S. Pope, Turbulent flows, Cambridge University Press, 2000.

[35] L. Zaichik, V. Alipchenkov, Statistical models for predicting pair dispersion and particle clustering in isotropic turbulence and their applications, New Journal of Physics 11 (2009) 103018.

[36] O. Simonin, L. Zaichik, V. Alipchenkov, P. Février, Connection between two statistical approaches for the modelling of particle velocity and concentration distributions in turbulent flow: The Mesoscopic Eulerian Formalism and the two-point probability density function method, Physics of Fluids 18 (12) (2006) 125107(1-9).

[37] E. Riber, Modeling turbulent two-phase flows using large-eddy simulation, Ph.D. thesis, INP Toulouse (2007).

URL http://ethesis.inp-toulouse.fr/archive/00000531/

[38] J. Dombard, Direct Numerical Simulation of non-isothermal dilute sprays using the Mesoscopic Eulerian Formalism, Ph.D. thesis, INP Toulouse (2011).

URL https://www . theses.fr/2011INPT0091

[39] P. Sierra, Modeling the dispersion and evaporation of sprays in aeronautical combustion chambers, Ph.D. thesis, INP Toulouse (2012).

URL https://tel.archives-ouvertes.fr/tel-00701105/

[40] M. Sabat, A. Larat, A. Vié, M. Massot, On the development of high order realizable schemes for the Eulerian simulation of disperse phase flows: a convex-state preserving Discontinuous Galerkin method, Journal of Computational Multiphase Flows 6 (3) (2014) 247-270.

[41] C. Chalons, M. Massot, A. Vié, On the Eulerian Large Eddy Simulation of disperse phase flows: an asymptotic preserving scheme for small stokes 
number flows, SIAM Journal on Multiscale Modeling and Simulation 13 (2015) 291-315.

[42] I. Celik, U. Ghia, P. Roache, C. Freitas, H. Coloman, P. Raad, Procedure of estimation and reporting of uncertainty due to discretization in cfd applications, J. Fluids Eng. 130 (2008) 078001.

[43] S. Descombes, M. Duarte, T. Dumont, F. Laurent, V. Louvet, M. Massot, Analysis of operator splitting in the non-asymptotic regime for nonlinear reaction-diffusion equations. Application to the dynamics of premixed flames, SIAM Journal on Numerical Analysis 52 (3) (2014) 1311-1334.

[44] B. van Leer, Towards the ultimate conservative difference scheme V. A second order sequel to Godunov's method, Journal of Computational Physics 32 (1) (1979) 101-136.

[45] C. Berthon, Stability of the MUSCL schemes for the Euler equations, Communications in Mathematical Sciences 3 (2) (2005) 133-157.

[46] A. Harten, P. D. Lax, B. van Leer, On upstream differencing and Godunovtype schemes for hyperbolic conservation laws, SIAM Review 25 (1) (1983) $35-61$.

[47] X. Zhang, C.-W. Shu, On positivity preserving high order Discontinuous Galerkin schemes for compressible euler equations on rectangular meshes, Journal of Computational Physics 229 (23) (2010) 8918-8934. 